\title{
Mental Health and Psychosocial Aspects of Corona Virus Disease (COVID-19) Outbreak in Ethiopia: Psychological Intervention for Public Psychological Crisis
}

\author{
Yordanos Yibeltal Yedemie \\ Department of Psychology, College of Education and Behavioral Sciences, Bahir Dar University, Bahir Dar, Ethiopia \\ Email address: \\ nuhaminyordanos@gmail.com \\ To cite this article: \\ Yordanos Yibeltal Yedemie. Mental Health and Psychosocial Aspects of Corona Virus Disease (COVID-19) Outbreak in Ethiopia: \\ Psychological Intervention for Public Psychological Crisis. International Journal of Psychological and Brain Sciences. \\ Vol. 5, No. 4, 2020, pp. 56-60. doi: 10.11648/j.ijpbs.20200504.11
}

Received: July 22, 2020; Accepted: August 17, 2020; Published: September 8, 2020

\begin{abstract}
The unpredictable COVID-19 occurrence has the potential of adversely affecting the mental wellbeing on individual and public level. At present all efforts are listening carefully on the understanding of epidemiology, clinical diagnosis and prognosis, frustrate the spread of the virus, and challenges of worldwide physical condition, while crucially significant mental wellbeing has been unnoticed in this attempt. This analysis is to assess past outbreaks to know the degree of adverse effects on Psychological panic, mental wellbeing, psychological crisis intervention, and mental health management strategy. COVID-19 is leading to severe psychosocial panics and comprising mental health marking a secondary health concern in globe. Internationally implementing preventive and controlling measures, and humanizing coping and resilience are demanding factors; modified lifestyle; symptoms, transmission, prevention and treatment; marginalization and hostility and universal collision on education, health, and climate are some of the risk factors to make worse further panics.
\end{abstract}

Keywords: Corona Virus Outbreak, Mental Health, Ethiopia, Psychological Crisis Intervention, Psychosocial Aspects, Public Mental Health Crisis

\section{Introduction}

Corona virus disease, a new strain belonging to the family of corona viruses which includes the Severe Acute Respiratory Syndrome (SARS)-CoV and the Middle East Respiratory Syndrome (MERS)-CoV, was first isolated in January 2020 by Chinese scientists, in Wuhan, Hubei province, China [1]. The outbreak strike Wuhan in late December 2019, when a large number of patients existing with pneumonia of unidentified etiology [1]. This new virus targets the respiratory system and the symptoms range from uncomplicated clinical manifestations such as dry cough, shortness of breath, sore throat, and fever to numerous fatal complications including cruel bilateral pneumonia, Acute Respiratory Distress Syndrome, septic shock, and eventually multi-organ failure. The epidemic, as confirmed by the World Health Organization on March 11, 2020, has caused havoc globally, attacking more than 169 countries.

With the arrival of Corona virus an unchartered territory in
Ethiopia on February, 2020, country is on high watchful and has reinforced partial lockdown. There are over eight million $(8,199,838)$ confirmed cases with more than half million deaths worldwide and in Ethiopia corona virus totally has risen to 3,759 confirmed cases with more than 63 deaths as of 1 st of June, 2020. The most horrifying hit countries (China, United States, Italy, Spain, Iran, France, United Kingdom, and Germany) are growing their efforts to handle the virulent disease through collective public health intervention measures. Even though WHO has labeled the outbreak the pandemic and Europe the epicenter of corona virus, Ethiopia is relatively less effected country viewed corona virus as the least of their problems, perhaps both by the public.

During the occurrence of deadly disease COVID-19, psychological crisis intervention for affected, suspected, vulnerable, and at-risk patients, care-givers, families, staff, and the general public, is immediately needed for fitting prevention of immeasurable hazards from secondary mental health catastrophe. The argument of psychological disaster intervention is to manage the side-and aftereffects on 
psychosocial aspects of an infectious disease and attempt to reduce psychological collision with timely assessment and management of prevention and control [1-8]. The two-O's prolonged approach (onsite and online) is essential to realize psychological crisis intervention. The present suggestions are presented as a temporary emergency proposal in an exceptional circumstance such as the widespread pandemic outbreak of COVID-19.

Different professionals have advised people to wait in selfisolation and quarantined but the essential restrictions would have short and potentially long term detrimental impact on psychological wellbeing. Ethiopia being a collectivistic culture extremely dependent on socialization has been serious towards self-isolation, social-distancing and quarantine and are unwillingly dealing with emotional, psychological, behavioral and social impacts of this crisis' indecision and unpredictability. The psychological impact of quarantine includes Posttraumatic Stress Disorder (PTSD), Obsessive Compulsive Disorder (OCD), Confusion, disturbance, bewilderment as well as frustration. This study highlighted the value of appropriate guideline of the COVID19, information and provision of resources during quarantined for better well-being. In that situation, reframing the situation as an opportunity to improve the psychological health, mindfulness and personal growth from side to side personal activities could become a resilient and protective issue against the psychological impact of corona virus. Anxiety frequently stemmed from the awareness of unmanageable events, consequently focusing on the controlled actions (change what you can) such as humanizing hygiene, healthy diet, exercising, sleeping, introspection, meditation, practicing minimalism, painting, composing, movement dancing, learning instruments, acquiring languages, knitting, gardening, cooking, reading books, listening songs, watching movies, playing games and journaling the personal observation and experience during the period of corona virus any factor that could offer resilient and protective strategies to handle with stress, anxiety, and panic should be practiced. Whereas government and health departments manage physical pandemic, experts (mental health practitioners, counseling psychologists, psychotherapists, social workers, Psychiatrist) could manage psychological health well-being and psychological factors of the various risks, resilience and recovery predictors. Mental health practitioners could offer online and onsite support through counseling, psychotherapy and direct them to reputable sources of information to avoid misunderstanding.

Previous studies on the infectious outbreaks of SARS, HIV/AIDs, MERS and Ebola discovered the severity of emotional distress not only in the general public but also among many therapeutic practitioners and law enforcement agents who faced PTSD, depression, anxiety, exhaustion and burnout at the onset, during and even after the outbreak of such epidemics. The case of COVID-19 poses more significant mental health deterioration since therapeutic practitioners and nurses are equally vulnerable to the infectious transmitting disease due to inadequate personal protective equipment, burnout, exhaustion, frustration, hopelessness, discrimination, isolation, patients with negative emotions and lack of contact with their families (10-15).

This worldwide public health concerns the role and responsibility of health workers, international impact of infection, impact of economic activities on travel and trade restrictions, and equitable care of public welfare and individual rights during the spread of pandemics. Psychological health effects could be minimized by avoiding excessive exposure to COVID-19 social media coverage, maintaining optimistic lifestyle and compassionately reassuring others as well. Resilience training programs for health staff, law enforcement agents and general public to handle with the after effects of the pandemic of this severity and intensity should be introduced: (a) family-work life balance; (b) reliable, genuine and timely incorporated information about the infectious disease and its consequences on psychological health; (c) teaching and preparing communities for pandemics and epidemics in the future; and (d) and validating and valuing frontline's staff's contribution (13-16).

\section{Methods}

This critical review is to evaluate past outbreaks to understand the epidemiology, cause, scientific symptoms and diagnosis, psychological wellbeing, psychological crisis intervention/prevention, management and future perspective of COVID 19 researcher findings. The study provinces, dates of publication, study areas, authors' affiliations, and methodological approach, MEDLINE, EMBASE, PubMed and Scopus databases were included for relevant articles. All the findings and statements in this review regarding the outbreak are based on published information as listed in the references.

\section{Results and Discussion}

Mental health services, facilities and specialized psychiatric treatment teams including psychologists, psychiatrists and psychiatric nurses should be recognized to address psychological health concerns in the general public. Individuals and communities could deliberately cultivate resilience, healthy coping strategies, mindfulness and wellbeing. The potential for resilience, coping, mindfulness and well-being are neither unique traits that one possess (or not) nor outcomes of absence of posttraumatic stress. The capacity for resilience is a process of adaptation. Coping mechanism is a learned pattern of behavior which one develops over the period of time. Mindfulness is the psychological process of intentionally bringing one's attention in the present moment which one develops with practice. Well-being is the experience of being comfortable in their state of affairs. These all are processes and they can be acquired with practice. Resilience, coping, mindfulness and well-being are not single dichotomous outcomes measuredstrengthening these processes intentionally reinforced 
practice by experiencing and learning dynamically.

Ethiopia must issue guidelines for emergency psychological crisis intervention for public health emergencies affected by COVID-19. Psychiatric and psychological institutions should operate platforms to make stronger mental wellbeing initiatives and offer psychological guidance to quarantined patients, families, self-isolated people, health-care workers of therapeutic and social service personnel in hospitals, laboratories, field, and in quarantine. Psychological crisis intervention, during and after the outbreak period, must focus on the practical realization of intervention. The challenge which psychological crisis intervention is encountering includes the establishment of psychological intervention teams throughout lockdown and personal health risks [3]. Furthermore, after the assessment of psychological health states of individuals affected by COVID-19 would remain confounded in the physical health unit, and even after the reduction of the pandemic, patients cannot be directly transferred from hospital to counseling psychological intervention units. During the vulnerability and defenselessness of the individual's conditions prior during and after the COVID-19, professionally experienced and standardized well trained mental health professionals, counselors, psychologists, practicing psychotherapists, psychiatrists, and psychiatrist nurses who are recognizable with the complicated case structures and work procedure is a essential principle in dealing with emotional distress and public psychological health emergencies caused by the pandemics, whereas inexperienced psychologists, untrained fresh graduates, non-practicing psychology teachers at academic institutes, certification/diploma holders or any other unwarranted personnel-until trained who are not always familiarized with the psychological crisis might have adverse effects in the existing pandemic situation which eventually would decrease the effectiveness of the entire intervention program [4] This situation could become worsen because of the officially un-established official approval, registration and licensure requirements for counseling psychologists, psychotherapists, counselors and mental health practitioners [5]. This situation can be resolved through strengthening personnel training, and optimizing institutional and management policies. Psychological crisis intervention would be implemented and adapted on a) quarantined patients with confirmed/suspected infection, b) self-isolated individuals susceptible to mental health concerns, c) health and related personnel directly in contact with patients, d) family caretakers of the patients, e) unwilling groups like older people, children, and pregnant women, and f) general public. Psychological crisis intervention should be based on the comprehensive assessment of risk factors for a standardized psychological/mental health management. Like major pandemic outbreak would have unenthusiastic effects on physical and psychological health of individuals and society [2, 6 \& 7], for example, psychological issues, psychological distress, grief and bereavement, deliberate or unintentional hurt to relatives, loss/separation from family, self-injury, shame, guilt, helplessness, posttraumatic stress symptoms, addiction or substance use, medical mistrust and inclination towards conspiracies, panic attacks, stress, anxiety, depression, loneliness, suicidal ideation, mood problems, sleep problems, worry, denial, boredom, ambivalence, uncertainty, frustration, anger, fear, stigmatization, marginalization, xenophobia, mass hysteria, socioeconomic status, and other psychological health concerns would require pre-established coalition to mobilize resources for effective intervention, management and preventive measures for affected individuals. Psychiatrists and practicing psychologists could offer a balanced perspective to improve the knowledge, attitude and Misinfodemics: a) teach people about the common psychological reactions, measures and responses, b) give confidence healthy activities during self-isolation, c) optimize accessible health-care, d) and empower healthcare and volunteer personnel.

1) First, psychological intervention technical support team established by health authorities at regional and national levels should deliver psychological intervention, and mental health support.

2) Second, accurate coordination and regular communication about the COVID-19 should be provided by health authority and psychosocial response teams to eliminate sense of uncertainty.

3) Third, ensure sound mental health through psychological counseling using government sanctioned hotline teams.

4) Fourth, patients with diagnosed corona virus and health care professionals should receive screening and counseling by professional mental health practitioners.

However, in low and middle income countries like Ethiopia which has low rate of psychological health service utilization and hardly active online psychological health services and inaccessibility of digital technology and health service for individuals with low socioeconomic status could be a risk factor in online psychological health service which should be considered. But, the Ethiopia COVID-19 Emergency recruitment agents besides the above activates should be focused on:

1) Health Supplies and Equipment

2) Preparedness, Capacity Building and Training

3) Community discussions and information outreach

4) Quarantine, Isolation, and Treatment Centers establishment

5) Establishing and equipping isolation centers with health supplies and furniture and network installation

6) Psychosocial crisis intervention teams should be established

7) Humanitarian partners are prioritizing life-saving activities and shifting resources to COVID -19 responses.

8) Site management is established at different quarantine sites

9) In collaboration with the Ministry of Education, the 
Education Cluster developed the 'Education Preparedness and Response Plan for COVID-19'. Ethiopia curriculum-based lessons through radio are expected to be rolled-out.

10) In support to COVID-19 response, boarding schools could be used as temporary treatment centers in the event that hospitals are overwhelmed.

11) Encouraging and supporting voluntary team workers.

\section{Conclusion and Recommendation}

I suggest that the psychological crisis intervention should be active, adapted to suit different stages of the epidemic, i.e., during and after the outbreak. During the outbreak, psychologist professionals should actively participate in the overall intervention process for the COVID-19, so that the mental health and psychosocial response can be mobilized in a timely manner. Specifically, psychological crisis interventions should be integrated into the treatment of pneumonia and blocking of the transmission routes. In this stage, psychological crisis intervention should include two simultaneous activities: (1) intervention for fear of disease, carried out mostly by physicians and assisted by psychologists; (2) intervention for complicatedness in adaptation, largely by mental health professionals and psychologists. Among them serious mental and psychological problems must be managed by psychiatrists and psychologists. This manuscript marks the need of establishment of guidelines for psychological crisis intervention effective immediately in Ethiopia.

\section{Author' Contributions}

The author conceived and designed the initial study and contributed to the conceptualization and design of the study. The author contributed to the development of the selection criteria, the risk of a bias assessment strategy, and data extraction criteria.

\section{Conflict of Interests}

I declare no competing interests.

\section{Ethical Approval}

Not required.

\section{References}

[1] Wang C, Horby PW, Hayden FG, Gao GF: A novel coronavirus outbreak of global health concern. Lancet. 2020, 395: 470-473. 10.1016/S0140-6736(20)30185-9.

[2] Kang L, Li Y, Hu S, et al. The mental health of medical workers in Wuhan, China dealing with the 2019 novel coronavirus. Lancet Psychiatry 2020; published online Feb 5. https://doi.org/10.1016/S2215-0366(20)30047-X.

[3] Zandifar A, Badrfam R. Iranian mental health during the COVID-19 epidemic. Asian J. Psychiatr 2020; 51: 101990. https://doi.org/10.1016/j.ajp.2020.101990.

[4] Bai Y, Lin C-C, Lin C-Y, Chen J-Y, Chue C-M, Chou P. Survey of stress reactions among health care workers involved with the SARS outbreak. Psychiatr Serv 2004; 55: 1055-57.

[5] Yao H, Chen J-H, Xu Y-F. Rethinking online mental health services in China during the COVID-19 epidemic. Asian J. Psychiatr 2020; 102015. https://doi.org/10.1016/j.ajp.2020.102015.

[6] Banerjee D. The COVID-19 outbreak: Crucial role the psychiatrists can play. Asian J. Psychiatr 2020; 102014. https://doi.org/10.1016/j.ajp.2020.102014.

[7] Brooks SK, Webster RK, Smith LE, Woodland L, Wessely S, Greenberg N, Gideon, JR. The psychological impact of quarantine and how to reduce it: rapid review of the evidence. The Lancet. 2020; 395: 10227.

[8] Centers for Disease Control and Prevention (CDCP) (2020). Coronavirus disease 2019 (COVID-19) Retrieved on March 26, 2020 from https://www.cdc.gov/coronavirus/about/index.html.

[9] Legido-Quigley, H., Mateos-García, J. T., Campos, V. R., Gea-Sánchez, M., Muntaner, C., y McKee, M. (2020). The resilience of the Spanish health system against the COVID-19 pandemic. https://doi.org/10.1016/S2468-2667(20)30060-8.

[10] Yao H, Chen JH, Xu YF. Patients with mental health disorders in the COVID-19 epidemic. 2020; 7: e21. [Links].

[11] Verity, R., Okell, L. C., Dorigatti, I., Winskill, P., Whittaker, C., Imai, N.,... Ferguson, N. (2020). Estimates of the severity of COVID-19 disease.

[12] Abdulahi H, Mariam DH, Kebede D. Burden of disease analysis in rural Ethiopia. Ethiop Med J. 2001 Oct; 39 (4): 271-81. PubMed | Google Scholar.

[13] Alem A, Araya M, Melaku Z et al. Mental distress in medical students of Addis Ababa University. Ethiop Med J. 2005; 43 (3): 159-166. PubMed | Google Scholar.

[14] Liu S, Yang L, Zhang C, Xiang YT, Liu Z, Hu S, et al. Online Mental Health Services in China During the COVID-19 Outbreak. Lancet Psychiatry. 2020 : s2215-0366. [PubMed: 32085841].

[15] Demyttenaere K, Bruffaerts R, Posada-Villa J, Gasquet I, Kovess V, Lepine JP et al. (2004). Prevalence, severity, and unmet need for treatment of mental disorders in the World Health Organization World Mental Health Surveys. JAMA. PubMed | Google Scholar.

[16] Donna Tedstone Doherty, Rosalyn Moran, Yulia Kartalova O'Doherty (2013). Psychological distress, mental health problems and use of health services in Ireland. HRB Research Series. http://www.ucd.ie/issda/static/documentation/hrb/hrbpsychreport.pdf. 


\section{Biography}

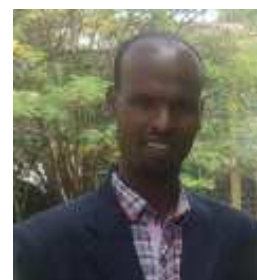

Yordanos Yibeltal Yedemie is BA holder in Psychology and Sociology and an MA holder in counseling psychology. I am currently Assistance Professor and Researcher in Bahr Dar University, Ethiopia. I do have eight article publications. I have been teaching Counseling \& Social psychology, Rehabilitation \& HIV/AIDs counseling, Marriage psychology and research courses for 14 years in HEIs. Besides, I was Dean of Educational and Behavioral Science College for 4 years in Woldia University. Now, I am department head of Psychology in Bahir Dar University. My main research interests are psychosocial issues, health issues, and educational change initiatives and related areas. 